\title{
ETV6 germline mutation - a risk for ALL
}

Hereditary predisposition is generally not considerered as a risk factor for the development of acute lymphoblastic leukaemia (ALL), the most-common cancer in children. Now, a study led by Jun Yang, shows that the presence of a familial component in ALL pathogenesis is much greater than it was previously believed.

Yang and colleagues identified a family with an unusual clustering of childhood ALL. With the hypothesis that this family had a genetic predisposition to developing ALL, whole-exome-sequencing analysis was performed for all family members. Among the nonsilent variants identified in patients with ALL from this family, priority was given to a novel ETV6 variant. The reason for focusing on this variant was that somatic mutations in ETV6 have already been linked to the development of haematological malignancies.

To confirm this association, targeted next-generation sequencing of ETV6 was performed in germline DNA samples from 4,405 children with ALL. In this large cohort, 31 rare ETV6 variants were identified that were potentially related to ALL predisposition. In children harbouring these mutations, ALL had distinct clinical features, such as older age at diagnosis, which indicate a unique mechanism of leukaemia pathogenesis related to these ETV6 variants.

The results of this study revealed that, although rare, the genetic predisposition for ALL does not occur only in exceptional cases. The authors estimate that approximately $1 \%$ of patients with childhood ALL potentially carry highly penetrant ETV6 variants. These findings provide a strong rationale for including family history examination as part of the standard approach to treating paediatric ALL. As Yang indicates, "guidelines are needed for the clinical interventions and surveillance of individuals with inherited predisposition variants."

Diana Romero

ORIGINAL ARTICLE Moriyama, T. et al. Germline genetic variation in ETV 6 and risk of childhood leukaemia: a systematic genetic study. Lancet Oncol. doi:10.1016/S1470-2045(15)00369-1 